\title{
Anti-inflammatory and Anti-infectious Dietary Paradigms May Be Crucial for Visceral Weight Reduction
}

\author{
Dong-Mei Chen ${ }^{1}$, Meng-Le Zhang ${ }^{2}$, Zhu-Qing Shi ${ }^{3}$, Chang-Qing Li ${ }^{1}$, Qi Wang ${ }^{4}$, \\ Jian-Ping Song ${ }^{1}$, Qin $\mathrm{Xu}^{1}, \mathrm{He} \mathrm{Li}^{2}$ and Qing-Ping Zeng ${ }^{1 *}$ \\ ${ }^{1}$ Institute of Tropical Medicine, Guangzhou University of Chinese Medicine, Guangzhou, China, ${ }^{2}$ School of Basic Course, \\ Guangdong Pharmaceutical University, Guangzhou, China, ${ }^{3}$ Science and Technology Industrial Park, Guangzhou University \\ of Chinese Medicine, Guangzhou, China, ${ }^{4}$ Institute of Clinical Pharmacology, Guangzhou University of Chinese Medicine, \\ Guangzhou, China
}

Keywords: obesity, weight loss, inflammation, infection, nutritional immunology

OPEN ACCESS

Edited by:

Pinyi Lu,

Biotechnology HPC Software Applications Institute (BHSAl),

United States

Reviewed by:

Manuela Del Cornò,

Istituto Superiore di Sanità (ISS), Italy

*Correspondence:

Qing-Ping Zeng

qpzeng@163.com

Specialty section:

This article was submitted to

Nutritional Immunology,

a section of the journa

Frontiers in Immunology

Received: 03 November 2018

Accepted: 18 February 2019

Published: 08 March 2019

Citation:

Chen D-M, Zhang M-L, Shi Z-Q, Li C-Q, Wang Q, Song J-P, Xu Q, Li H

and Zeng Q-P (2019)

Anti-inflammatory and Anti-infectious Dietary Paradigms May Be Crucial for

Visceral Weight Reduction.

Front. Immunol. 10:422.

doi: 10.3389/fimmu.2019.00422
Based on body mass index (BMI), body weight has been classified into overweight (25-29.9 $\left.\mathrm{kg} / \mathrm{m}^{2}\right)$, obesity $\left(\geq 30 \mathrm{~kg} / \mathrm{m}^{2}\right)$, and severe obesity $\left(\geq 40 \mathrm{~kg} / \mathrm{m}^{2}\right)(1)$. BMI $>30$ has been classified as a disease state by the American Medical Association (AMA) (2). According to the World Health Organization (WHO), the worldwide prevalence of obesity almost tripled between 1975 and 2016. Overall, 1.9 billion (39\%) and 650 million (13\%) of adults aged 18 years and over were overweight and obese, respectively, in 2016 (3). From 2007-2016 in the US, middle-aged obese adults (40-59 years old) were more prevalent than obese young adults (4). These statistical data identified middle-aged obese adults as a high-risk population vulnerable to obesity-related metabolic syndrome.

Visceral obesity, also known as abdominal, central, or ectopic obesity, was defined as a waist circumference $\geq 102 \mathrm{~cm}$ in men and $\geq 88 \mathrm{~cm}$ in women (5), or as a waist-to-hip ratio $>0.9$ for men and $>0.85$ for women (6). Recently, $50 \%$ of men and $70 \%$ of women among US adults aged from 50 to 79 years were diagnosed with visceral obesity (7), which closely correlated with insulin resistance, type 2 diabetes, and cardiovascular disease (8).

Neither BMI-defined obesity nor visceral obesity provides guidance on how to reduce weight in young or elderly populations because the etiology of obesity in these populations is uncertain. We suggest that weight loss would be more practical if obesity was simply classified into inflammatory and non-inflammatory subtypes, regardless of race and gender. We believe that a shift from non-inflammatory obesity to inflammatory obesity may be aging-dependent and adipose depot-specific. As such, subcutaneous obesity in young individuals, with the exception of extreme cases of adolescent obesity, would be generally non-inflammatory. In contrast, visceral obesity frequently seen in elderly individuals is more likely to be inflammatory. However, inflammatory obesity can also occur in young adults, adolescents, and children (see below).

This classification of obesity subtypes was suggested because we believe that immunosurveillance determines conversion from non-inflammatory to inflammatory obesity subtype. Aging-driven immunosenescence deteriorates innate and adaptive immunity, leading to compromised elimination of pathogenic and opportunistic infections (9). Non-inflammatory obesity equates to metabolically-healthy obesity, whereas inflammatory obesity equates to metabolically-unhealthy obesity (10), with the latter associated with increased risk of cardiovascular disease-related mortality (11). 
In general, visceral obesity is accompanied by infiltration of activated macrophages and other immunocompetent cells, as demonstrated by increased area, density, and presentation of inflammatory markers in abdominal intramuscular adipose tissue (12). Adipose inflammation is likely induced by either the bacterial endotoxin lipopolysaccharide (LPS) (13), fatty acids (14), or ceramides (15).

Visceral obesity could be conveniently measured using a bioelectrical impedance analysis (BIA)-based electric meter (16). Accordingly, lower basal metabolic rate (BMR) and lower body water rate (BWR), which can be measured using these meters, might be indicators of visceral obesity, although this association requires further elucidation. However, inflammation causes mitochondrial dysfunction, resulting in disruption of fatty acid oxidation, and decreased ATP and $\mathrm{H}_{2} \mathrm{O}$ production. For example, age-dependent obesity was associated with decreased mitochondrial complex IV activity, resulting in reduced fatty acid oxidation and subsequent adipocyte hypertrophy (17).

Therefore, inflammatory obesity in immunocompromised middle-aged and elderly adults is assumed to originate from gut dysbiosis, colon damage, LPS leakage, and mitochondrial depletion. This subtype of obesity, characterized by less fatty acid degradation, may be ameliorated by anti-infection and antiinflammatory treatment.

In contrast, non-inflammatory obesity in adolescents or children with competent immune systems may simply result from excessive food intake and inadequate energy expenditure. This subtype of obesity is characterized by increased fatty acid and fat synthesis, and may be best treated by calorie restriction (CR), intermittent fasting (IF), exercise training, or other weightreducing procedures.

\section{GUT DYSBIOSIS INDUCES COLON DAMAGE AND ENDOTOXIN LEAKAGE}

Recent studies suggested that sensitivity of gut microbiota to host genetic and dietary influences contribute to risk of development of obesity and related metabolic disorders (18). A previous study showed that $37.6 \%$ of obese children presented with small intestine bacterial overgrowth (SIBO). Non-alcoholic fatty liver disease (NAFLD), hypertension, and metabolic syndrome accounted for 59.5, 23.4, and $44.6 \%$ in the SIBO positive group, compared with 10.2, 5.1, and $9 \%$ in the SIBO negative group (19), implying that intestinal infection was a major contributor to NAFLD, hypertension, and metabolic syndrome in obese children.

A common ingredient in livestock and poultry products, chondroitin sulfate (CS), increases abundance of Bacteroides thetaiotaomicron, a species of sulfatase-secreting bacteria that degrades mucins to supply sulfate to Desulfovibrio piger, a species of sulfate-reducing bacteria (20). Heme, a rich component in red meat, contributes to increased abundance of Akkermansia muciniphila, a species of mucus-degrading bacteria, and further facilitates aberrant colon epithelial proliferation through consumption of mucins (21). Beneficial or harmful effects of A. muciniphila have been shown to be abundancedependent. Colon integrity and barrier function were reinforced by adequate mucin-consumer residence, but compromised by excessive mucin consumption (22).

High-fat diet (HFD) led to increased secretion of bile acids (BAs), followed by alterations in microbial compositions. Feeding mice BAs with a normal diet induced an obese phenotype, similar to that seen in HFD-fed mice. Interruption of BA biosynthesis attenuated HFD-shaped plasticity of the gut microbiome (23). HFD increased oxidative stress and disrupted intestinal gap junction proteins, increased membrane permeability, and contributed to endotoxemia, inflammation, and intestinal tumorigenesis (24).

\section{GUT DYSBIOSIS TRIGGERS ADIPOSE INFLAMMATION AND MITOCHONDRIAL DYSFUNCTION}

Mice fed an obesogenic but non-inflammatory diet developed metabolically-healthy obesity, but fed a Paigen diet developed metabolically-unhealthy obesity. This study showed that $\mathrm{T}$ lymphocyte infiltration occurred in response to obesogenic and Paigen diets, but $\mathrm{CD}^{+}{ }^{+}$and $\mathrm{CD}^{+}$cells were increased only in Paigen-fed mice, and showed increased expression of interleukin 1 (IL-1), IL-4, IL-6, IL-17, and interferon $\gamma$ (IFN- $\gamma$ ). Accordingly, the colon-destroying bacteria Bacteroidia, Deltaproteobacteria, and Verrucomicrobia dominated the gut lumen of mice fed a Paigen diet (25). These results provided direct evidence supporting classification of obesity into inflammatory and noninflammatory subtypes.

As brown adipose tissue (BAT), which contains a large amount of mitochondria, converts to white adipose tissue (WAT), which contains relatively fewer mitochondria, many degenerating mitochondria containing activated inflammasome NLR family pyrin domain containing 3 (NLRP3) were observed in whitened adipocytes (26). Upon activation of hypoxia-inducible factor $1 \alpha($ HIF- $1 \alpha)$, palmitate-induced pro-inflammatory cytokine IL$1 \beta$ and macrophage Janus kinase-p38 mitogen-activated protein kinase (JNK-p38 MAPK) were upregulated and activated (27). Activity and assembly of mitochondrial complex IV were repressed in adipocytes of middle-aged mice and human visceral adipose tissue in a HIF- $1 \alpha$-dependent manner (28).

When mitochondrial density becomes scattered and dysfunctional as an outcome of inflammation, fatty acids from fat digestion cannot be appropriately converted to adenosine triphosphate (ATP), $\mathrm{CO}_{2}$, and $\mathrm{H}_{2} \mathrm{O}$. Because of mitochondrial dysfunction, inflammatory obesity should be characterized by incomplete fatty acid oxidation. Indeed, knockout of the anti-inflammatory cytokine IL10 resulted in an inflammatory state, which lowers body temperature in newborns due to impaired UCP1-dependent mitochondrial respiration in BAT (29). As further evidence, anti-inflammatory effects induced by antibiotics or non-steroidal anti-inflammatory agents (NSAIDs), such as aspirin, showed better weight-reducing effects (30).

In turn, water deficits increase serum levels of antidiuretic hormone $(\mathrm{ADH})$, vasopressin, and glucocorticoids, resulting 
in activation of serum- and glucocorticoid-inducible kinase 1 (SGK1), adipose deposition, and obesity-related disorders. Accordingly, water insufficiency also augmented nuclear factor of activated T-cells 5 (NFAT5) effects that could stimulate SGK1 activation and induce fat deposition (31).

\section{AN ANTI-INFLAMMATORY HIGH-FAT AND LOW-CARBOHYDRATE DIET MIGHT BE EFFECTIVE FOR ADIPOSE WEIGHT LOSS}

Anti-obesity effects exerted by a high-fat and low-carbohydrate ketogenic diet (KD) have been extensively debated (32-34) because the ketogenic diet has been shown to contribute to gut dysbiosis. Recent clinical trial data have indicated that KD represents a healthy diet for weight loss (35). A meta-analysis of 13 randomized controlled trials over 1 year indicated that volunteers on a very low carbohydrate $\mathrm{KD}$ tended to lose more weight than those on a low-fat diet in five trials (36). In an 8week randomized trial that included 34 obese men and women aged 60 to 75 , those on the KD lost $9.7 \%$ of body fat, while those on a low-fat diet lost only $2.1 \%$ of body fat, and those on the $\mathrm{KD}$ lost three times more visceral adipose weight than those on a low-fat diet (37).

In a 3-month prospective observational study of glucose transporter 1 (GLUT1) deficiency syndrome, a disorder in which individuals cannot utilize glucose, KD significantly increased Desulfovibrio spp., a bacterial group linked to gut mucosa inflammation and animal fat consumption (38). Akkermansia and Parabacteroides enriched by KD provided protection from seizure in a mouse seizure model (39). Interestingly, KD reversed overgrowth of A. muciniphila and elicited an anti-microbiallike effect in mice (40). $\beta$-hydroxybutyrate, a major ketone body (KBs) derived from fatty acid degradation in the liver, could block NLRP3-mediated inflammation and attenuate IL$1 \beta$ secretion (41), implying that $\mathrm{KD}$ might modulate host inflammatory responses through high fat content leading to inflammation and $\beta$-hydroxybutyrate production leading to antiinflammatory effects.

The low carbohydrate effects of $\mathrm{KD}$ could mimic $\mathrm{CR}$ to activate adenosine monophosphate-activated protein kinase (AMPK) after an increase in AMP, leading to activation of silent mating type information regulation 2 homolog-1 (SIRT1) after an increase in nicotinamide adenine dinucleotide $\left(\mathrm{NAD}^{+}\right)$. AMPK and SIRT1 cooperatively activate peroxisome proliferator activated receptor $\gamma$ coactivator $1 \alpha$ (PGC-1 $\alpha)$ to induce mitochondrial biogenesis, fatty acid oxidation, and adipose weight loss $(42,43)$. Crosstalk between KD-mediated histone deacetylase (HDAC) inhibition and mechanistic target of rapamycin catalysis subunit 1 (mTORC1) signaling has been shown to contribute to lifespan extension in mice $(44,45)$.

Peroxisome proliferator activated receptor $\alpha(\operatorname{PPAR} \alpha)$, a key transcription factor in regulation of ketogenesis, has been shown to participate in signaling driven by AMPK, PGC- $1 \alpha$, and mTORC1. PPAR $\alpha$ also induced the hormonal mediator fibroblast growth factor 21 (FGF21) to activate hepatic lipolysis and ketogenesis (46). Therefore, a KD-like diet can prompt fatty acid conversion to anti-inflammatory KBs in the liver when glucose supply is insufficient.

\section{AN ANTI-INFECTIOUS FIBER-RICH DIET MIGHT CONTRIBUTE TO ADIPOSE WEIGHT LOSS}

Gut microbial fermentation of vegetables and fruits produces short-chain fatty acids (SCFAs) including acetate, propionate, and butyrate. SCFAs contributed to a healthier gut microbial ecological system and ameliorated type 2 diabetes (47). Furthermore, butyrate protected mice against methioninecholine-deficient diet-induced non-alcoholic steatohepatitis (NASH) by improving gut barrier function, attenuating inflammation, and reducing endotoxin levels (48). Butyrate also activated G protein-coupled receptor 43 (GPR43) and suppressed insulin signaling in adipocytes, thereby inhibiting fat accumulation and promoting lipid metabolism (49). Hepatic mitochondria served as the main targets of butyrate in reversing insulin resistance and blocking fat accumulation in diet-induced obese mice (50). Therefore, fiber-rich diets have anti-infectious properties, and components of these diets can be fermented into anti-infectious SCFAs by gut bacteria.

Due to being structurally related, butyrate and $\beta$ hydroxybutyrate should be functionally redundant. A recent study demonstrated that several four-carbon organic molecules, including butyrate and $\beta$-hydroxybutyrate, favored energy expenditure and alleviated oxidative stress (51). Additionally, acetate showed anti-inflammatory and oxidative stressmodulating properties in different immune cells (52), suggesting that a diet that contains vinegar may help to prohibit bacterial overgrowth, maintain gut microbiota homeostasis, and contribute to adipose weight reduction.

\section{AN INNOVATIVE ADIPOSE WEIGHT-REDUCING DIET FOR MIDDLE-AGED OBESE ADULTS}

We strongly recommend a convenient and practical "farmerhunter" diet, or a modified fiber-rich KD, as an ideal weightreducing dietary option for middle-aged obese adults. This diet includes a high-fiber vegetarian breakfast and lunch (07:0019:00 for $12 \mathrm{~h}$ duration) supplemented with tea and coffee, and a KD-like carnivorous dinner (19:00-07:00 for $12 \mathrm{~h}$ duration) combined with wine or vinegar. First, carnivorous food (meat, fish, and sea food)-derived KBs prevent chronic inflammation and mimic CR to enhance mitochondrial biogenesis for effective fatty acid oxidation and energy expenditure (53). Second, vegetarian food (cereals, legumes, vegetables, and fruits)-derived SCFAs mitigate meat-induced gut opportunistic infection and maintain gut ecological homeostasis (54). Third, tea, coffee, and wine, rich in polyphenols, can serve as anti-oxidants or can be fermented to SCFAs to prevent infection (55). Finally, acetate in vinegar can mimic SCFAs to exert anti-infectious effects (56).

Our recommendation is that rice, bread, and other starchy foods must be consumed separately from meat, fish, and 


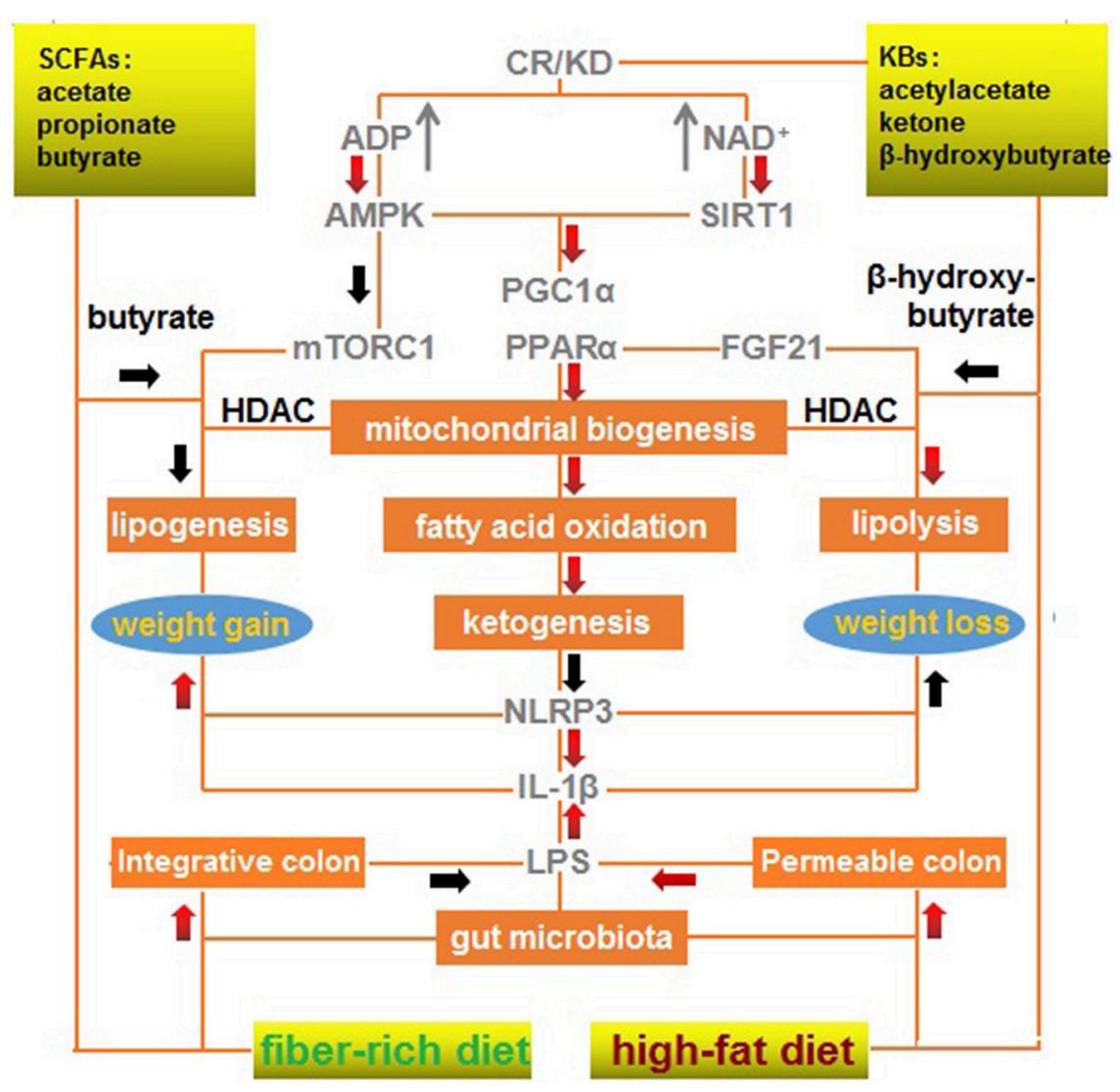

FIGURE 1 | A mechanistic outline of the interactions between a modified fiber-rich ketogenic diet with multiple signaling pathways associated with weight loss. A ketogenic diet mimics calorie restriction to improve mitochondrial function via ketone bodies, resulting in anti-inflammatory effects, and a fiber-rich diet maintains gut homeostasis via short-chain fatty acids, which exert anti-infectious effects. A red arrow represents positive regulation (upregulation/increase); and a black arrow represents negative regulation (downregulation/decrease). CR, calorie restriction; KBs, ketone bodies; KD, ketogenic diet; SCFAs, short-chain fatty acids.

seafood to avoid conversion of excess glucose to lipids. Without worrying about meat and oil-induced gut dysbiosis (21-24), those above described anti-inflammatory and anti-infectious dietary components should ensure sustainable availability of active and functional mitochondria for fatty acid metabolism and adipose weight loss (31-36).

\section{CONCLUSION}

Maintenance of gut microbiota homeostasis is the most critical factor in eliminating inflammatory obesogenic drivers, particularly with regard to gut opportunistic infection and endotoxin-triggered inflammation. SCFAs and KBs, which are structurally and functionally similar, are complementary in restoring gut homeostasis and rectifying mitochondrial dysfunction. An integrative signaling framework responsible for weight loss is summarized in Figure 1. This scheme illustrates a primary effect of $\mathrm{CR} / \mathrm{KD}$ on mitochondrial biogenesis, fatty acid oxidation, and ketogenesis, as well as concurrent effects of a fiberrich diet on the integrative colon and low-level LPS and effects of a high-fat diet on the permeable colon and high-level LPS.

Briefly, KD can mimic CR to activate AMPK, SIRT1, PGC$1 \alpha$, and PPAR $\alpha$ to enhance mitochondrial biogenesis, fatty acid oxidation, and ketogenesis. These processes can inhibit NLRP3 and IL-1 $\beta$ that promote weight gain and repress weight loss. Butyrate from a fiber-rich diet can mimic the effects of $\beta$ hydroxybutyrate produced by a high-fat diet to inhibit HDAC, promote lipolysis, and repress lipogenesis, resulting in mTORC1 inactivation and FGF21 activation.

\section{AUTHOR CONTRIBUTIONS}

Q-PZ wrote the manuscript. J-PS, QX, C-QL, and QW critically reviewed the manuscript. All authors read and approved the final version of the manuscript.

\section{FUNDING}

This work was supported by the National Natural Science Foundation of China (NSFC, No. 81273620 and 81774041 to Q-PZ, No. 81673861 to C-QL and 81873218 to J-PS).

\section{ACKNOWLEDGMENTS}

We thank Yan-Ping Chen and Li-Li Tan for their assistance in manuscript preparation. 


\section{REFERENCES}

1. Jensen MD, Ryan DH, Apovian CM, Ard JD, Comuzzie AG, Donato $\mathrm{KA}$, et al. $2013 \mathrm{AHA} / \mathrm{ACC} / \mathrm{TOS}$ guideline for the management of overweight and obesity in adults. Circulation. (2014) 129:S102-38. doi: 10.1161/01.cir.0000437739.71477.ee

2. Jarris PE. Obesity as disease: an opportunity for integrating public health and clinical medicine. J Public Health Manag Pract. (2013) 19:610-2. doi: 10.1097/PHH.0b013e3182a8f8c4

3. WHO. Fact Sheet on Obesity and Overweight. Available online at: http://www. who.int/topics/obesity/en/ (Accessed February 16, 2018).

4. Hales CM, Fryar CD, Carroll MD, Freedman DS, Ogden CL. Trends in obesity and severe obesity prevalence in US Youth and Adults by Sex and Age, 20072008 to 2015-2016. JAMA. (2018) 319:1723-5. doi: 10.1001/JAMA.2018.3060

5. Stephen N, Häring HU, Schulze MB. Metabolically healthy obesity: the lowhanging fruit in obesity treatment? Lancet Diabetes Endocrinol. (2017) 6:24958. doi: 10.1016/S2213-8587(17)30292-9

6. Yusuf S, Hawken S, Ounpuu S, Dans T, Avezum A, Lanas F, et al. Effect of potentially modifiable risk factors associated with myocardial infarction in 52 countries (the INTERHEART study): case-control study. Lancet. (2004) 364:937-52. doi: 10.1016/S0140-6736(04)17018-9

7. Li C, Ford ES, McGuire LC, Mokdad AH. Increasing trends in waist circumference and abdominal obesity among US adults. Obesity. (2007) 15:216-24. doi: 10.1038/oby.2007.505

8. Gabriely I, Ma XH, Yang XM, Atzmon G, Rajala MW, Berg AH, et al. Visceral fat. Diabetes. (2002) 51:2951-8. doi: 10.2337/diabetes.51.10.2951

9. Sellami M, Gasmi M, Denham J, Hayes LD, Stratton D, Padulo J, et al. Effects of acute and chronic exercise on immunological parameters in the elderly aged: can physical activity counteract the effects of aging? Front Immunol. (2018) 9:2187. doi: 10.3389/fimmu.2018.02187

10. Eckel N, Li Y, Kuxhaus O, Stefan N, Hu FB, Schulze MB. Transition from metabolic healthy to unhealthy phenotypes and association with cardiovascular disease risk across BMI categories in 90257 women (the Nurses' Health Study): 30 year follow-up from a prospective cohort study. Lancet Diabetes Endocrinol. (2018) 6:714-24. doi: 10.1016/S2213-8587(18)30137-2

11. Lavie CJ, Deedwania P, Ortega FB. Obesity is rarely healthy. Lancet Diabetes Endocrinol. (2018) 6:678-9. doi: 10.1016/S2213-8587(18) 30143-8

12. Vella CA, Allison MA. Associations of abdominal intermuscular adipose tissue and inflammation: the multi-ethnic study of atherosclerosis. Obes Res Clin Pract. (2018) 12:534-40. doi: 10.1016/j.orcp.2018.08.002

13. Clemente-Postigo M, Oliva-Olivera W, Coin-Aragüez L, Ramos-Molina B, Giraldez-Perez RM, Lhamyani S, et al. Metabolic endotoxinemia promotes adipose dysfunction and inflammation in human obesity. Am J Physiol Endocrinol Metab. (2018) 316:E319-32. doi: 10.1152/ajpendo.00277.2018

14. Lee YS, Kim JW, Osborne O, Oh DY, Sasik R, Schenk S, et al. Increased adipocyte $\mathrm{O} 2$ consumption triggers HIF- $1 \alpha$, causing inflammation and insulin resistance in obesity. Cell. (2014)157:1339-52. doi: 10.1016/j.cell.2014. 05.012

15. Xia JY, Holland WL, Kusminski CM, Sun K, Sharma AX, Pearson MJ, et al. Targeted induction of ceramide degradation leads to improved systemic metabolism and reduced hepatic steatosis. Cell Metab. (2015) 22:266-78. doi: 10.1016/j.cmet.2015.06.007

16. Peterson JT, Repovich WES, Parascand CR. Accuracy of consumer grade bioelectrical impedance analysis devices compared to air displacement plethysmography. Med Sci Spot Ex. (2007) 39:S373. doi: 10.1249/01.mss.0000274464.50186.ed

17. Li QOY, Soro-Arnaiz I, Aragones J. Age-dependent obesity and mitochondrial dysfunction. Adipocyte. (2017) 6:161-6. doi: 10.1080/21623945.2017.1297346

18. Caricilli AM, Saad MJ. Gut microbiota composition and its effects on obesity and insulin resistance. Curr Opin Clin Nutr Metab Care. (2014) 17:312-8. doi: 10.1097/MCO.0000000000000067

19. Belei O, Olariu L, Dobrescu A, Marcovici T, Marginean O. The relationship between non-alcoholic fatty liver disease and small intestinal bacterial overgrowth among overweight and obese children and adolescents. J Pediatr Endocrinol Metab. (2017) 30:1161-8. doi: $10.1515 /$ jpem-2017-0252
20. Rey FE, Gonzalez MD, Cheng J, Wu M, Ahern PP, Gordon JI. Metabolic niche of a prominent sulfate-reducing human gut bacterium. Proc Natl Acad Sci USA. (2013) 110:13582-7. doi: 10.1073/pnas.1312524110

21. Ijssennager N, Belzer C, Hooiveld GJ, Dekker J, van Mil SW, Müller M, et al. Gut microbiota facilitates dietary heme-induced epithelial hyper-proliferation by opening the mucus barrier in colon. Proc Natl Acad Sci USA. (2015) 112:10038-43. doi: 10.1073/pnas.1507645112

22. Wang Q, Huang SQ, Li CQ, Xu Q, Zeng QP. Akkermansia muciniphila may determine chondroitin sulfate ameliorating or aggravating osteoarthritis. Front Microbiol. (2017) 8:1955. doi: 10.3389/fmicb.2017.01955

23. Zheng X, Huang F, Zhao A, Lei S, Zhang Y, Xie G, et al. Bile acid is a significant host factor shaping the gut microbiome of diet-induced obese mice. BMC Biol. (2017) 15:120. doi: 10.1186/s12915-017-0462-7

24. Park MY, Kim MY, Seo YR, Kim JS, Sung MK. High-fat diet accelerates intestinal tumorigenesis through disrupting intestinal cell membrane integrity. J Cancer Prev. (2016) 21:95-103. doi: 10.15430/JCP.2016.21.2.95

25. Pindjakova J, Sartini C, Lo Re O, Rappa F, Coupe B, Lelouvier B, et al. Gut dysbiosis and adaptive immune response in diet-induced obesity vs. systemic inflammation. Front Microbiol. (2017) 8:1157. doi: 10.3389/fmicb.2017.01157

26. Kotzbeck P, Giordano A, Mondini E, Murano I, Severing I, Venema W, et al. Brown adipose tissue whitening leads to brown adipocyte death and adipose tissue inflammation. J Lipid Res. (2018) 59:784-94. doi: 10.1194/jlr.M079665

27. Snodgrass RG, Boß M, Zezina E, Weigert A, Dehne N, Fleming I, et al. Hypoxia potentiates palmitate-induced pro-inflammatory activation of primary human macrophages. J Bio Chem. (2016) 291:413-24. doi: $10.1074 /$ jbc.M115.686709

28. Soro-Arnaiz I, Li QOY, Torres-Capellini M, Melendez-Rodriguez F, Veiga S, Veys $\mathrm{K}$, et al. Role of mitochondrial complex IV in age-dependent obesity. Cell Rep. (2016) 16:2991-3002. doi: 10.1016/j.celrep.2016.08.041

29. de-Lima-Júnior JC, Souza GF, Moura-Assis A, Gaspar RS, Gaspar JM, Rocha $\mathrm{AL}$. Abnormal brown adipose tissue mitochondrial structure and function in IL10 deficiency. EBioMed. (2018) 39:436-47. doi: 10.1016/j.ebiom.2018.11.041

30. Zeng ZJ, Liu RX, Li AR, Guo JW, Zeng QP. How do structurally distinct compounds exert functionally identical effects in combating obesity? Front Pharmacol. (2018) 9:69. doi: 10.3389/fphar.2018.00069

31. Lang F, Guelinckx I, Lemetais G, Menander O. Two liters a day keep the doctor away? Considerations on the pathophysiology of suboptimal fluid Intake in the common population. Kidney Blood Press Res. (2017) 42:483-94. doi: $10.1159 / 000479640$

32. Astrup A, Meinert Larsen T, Harper A. Atkins and other low-carbohydrate diets: hoax or an effective tool for weight loss? Lancet. (2004) 364:897-9. doi: 10.1016/S0140-6736(04)16986-9

33. Bravata DM, Sanders L, Huang J, Krumholz HM, Olkin I, Gardner CD, et al. Efficacy and safety of low-carbohydrate diets: a systematic review. JAMA. (2003) 289:1837-50. doi: 10.1001/jama.289.14.1837

34. Brouns F. Overweight and diabetes prevention: is a low-carbohydratehigh-fat diet recommendable? Eur J Nutr. (2018) 57:1301-12. doi: $10.1007 / \mathrm{s} 00394-018-1636-y$

35. Abbasi J. Interest in the ketogenic diet grows for weight loss and type 2 diabetes. JAMA. (2018) 319:215-7. doi: 10.1001/jama.2017.20639

36. Bueno NB, de Melo ISV, de Olivaira SL, da Rocha Ataide T. Very-lowcarbohydrate ketogenic diet $v$. low-fat diet for long-term weight loss: a metaanalysis of randomised controlled trials. Br J Nutr. (2013) 110:1178-87. doi: $10.1017 /$ S000711451300548

37. Goss AM, Gower BA, Soleymani T, Stewart M, Fontaine K. Effects of an egg-based, carbohydrate-restricted diet on body composition, fat distribution, and metabolic health in older adults with obesity: preliminary results from a randomized controlled trial. FASEB J. (2017) 31:IB320.

38. Tagliabue A, Ferraris C, Uggeri F, Trentani C, Bertoli S, Giorgio V, et al. Short-term impact of a classical ketogenic diet on gut microbiota in GLUT1 deficiency syndrome: a 3-month prospective observational study. Clin Nutr ESPEN. (2017) 17:33-7. doi: 10.1016/j.clnesp.2016.11.003

39. Olson CA, Vuong HE, Yano JM, Liang QY, Nusbaum DJ, Hsiao EY. The gut microbiota mediates the anti-seizure effects of the ketogenic diet. Cell. (2018) 173:1728-41.e13. doi: 10.1016/j.cell.2018.04.027

40. Newell C, Bonhoeffer MR, Reiner RA, Hittel DS, Rho JM, Shearer J. Ketogenic diet modifies the gut microbiota in a murine model of autism spectrum disorder. Mol Autism. (2016) 7:37. doi: 10.1186/s13229-016-0099-3 
41. Youm YH, Nguyen KY, Grant RW, Goldberg EL, Bodogai M, Kim D, et al. The ketone metabolite $\beta$-hydroxybutyrate blocks NLRP3 inflammasome-mediated inflammatory disease. Nat Med. (2015) 21:263-9. doi: 10.1038/nm.3804

42. Bae HR, Kim DH, Park MH, Lee B, Kim MJ, Lee EK, et al. $\beta$ Hydroxybutyrate suppresses inflammasome formation by ameliorating endoplasmic reticulum stress via AMPK activation. Oncotarget. (2016) 7:66444-54. doi: 10.18632/oncotarget.12119

43. Wang DT, He J, Wu M, Li SM, Gao Q, Zeng QP. Artemisinin mimics calorie restriction to trigger mitochondrial biogenesis and compromise telomere shortening in mice. Peer J. (2015) 3:e822. doi: 10.7717/peerj.822

44. Roberts MN, Wallace MA, Tomilov AA, Zhou ZY, Marcotte GR, Tran D, et al. A ketogenic diet extends longevity and healthspan in adult mice. Cell Metab. (2017) 26:539-46. doi: 10.1016/j.cmet.2017.08.005

45. Newman JC, Covarrubias AJ, Zhao M, Yu X, Gut P, Ng CP, et al. Ketogenic diet reduces midlife mortality and improves memory in aging mice. Cell Metab. (2017) 26:547-57.e8. doi: 10.1016/j.cmet.2017. 08.004

46. Grabacka M, Pierzchalska M, Dean M, Reiss K. Regulation of ketone body metabolism and the role of PPAR $\alpha$. Intern J Mol Sci. (2016) 17:2093. doi: 10.3390/ijms17122093

47. Zhao LP, Zhang F, Ding X, Wu G, Lam YY, Wang X, et al. (2018). Gut bacteria selectively promoted by dietary fibers alleviate type 2 diabetes. Science. (2018) 359:1151-6. doi: 10.1126/science.aao5774

48. Ye JZ, Lv LX, Wu WR, Li YT, Shi D, Fang DQ, et al. Butyrate protects mice against methionine- choline-deficient diet-induced nonalcoholic steatohepatitis by improving gut barrier function, attenuating inflammation and reducing endotoxin levels. Front Microbiol. (2018) 9:1967. doi: 10.3389/fmicb.2018.01967

49. Kimura I, Ozawa K, Inoue D, Imamura T, Kimura K, Maeda T, et al. The gut microbiota suppresses insulin-mediated fat accumulation via the short-chain fatty acid receptor GPR43. Nat Commun. (2013) 4:1829. doi: $10.1038 /$ ncomms 2852

50. Miletta MC, Petkovic V, Ebel A, Amman RA, Fluck CE, Mullins PE. Butyrate increases intracellular calcium levels and enhances growth hormone release from ratanterior pituitary cells via the G-protein-coupled receptors
GPR41 and 43. PLoS ONE. (2014) 9:e107388. doi: 10.1371/journal.pone.01 07388

51. Christ S, Pirola L. Essential roles of four-carbon backbone chemicals in the control of metabolism. World J Biol Chem. (2015) 6:223-30. doi: 10.4331 /wjbc.v6.i3.223

52. Al-Harbi NO, Nadeem A, Ahmad SF, Alotaibi MR, AlAsmari AF, Alanazi WA, et al. Short chain fatty acid, acetate ameliorates sepsis-induced acute kidney injury by inhibition of NADPH oxidase signaling in $\mathrm{T}$ cells. Int Immunopharmacol. (2018) 58:24-31. doi: 10.1016/j.intimp.2018.02.023

53. Veech RL, Bradshaw PC, Clarke K, Curtis W, Pawlosky R, King MT. Ketone bodies mimic the life span extending properties of caloric restriction. IUBMB Life. (2017) 69:305-14. doi: 10.1002/iub.1627

54. Bach Knudsen KE, Lærke HN, Hedemann MS, Nielsen TS, Ingerslev AK, Gundelund Nielsen DS. Impact of diet-modulated butyrate production on intestinal barrier function and inflammation. Nutrients. (2018) 10:E1499. doi: 10.3390/nu10101499

55. Rothenberg DO, Zhou C, Zhang L. A review on the weight-loss effects of oxidized tea polyphenol. Molecules. (2018) 23:E1176. doi: 10.3390/molecules23051176

56. Shen F, Feng J, Wang X, Qi Z, Shi X, An Y, et al. Vinegar treatment prevents the development of murine experimental colitis via inhibition of inflammation and apoptosis.J Agric Food Chem. (2016) 64:1111-21. doi: $10.1021 /$ acs.jafc. 5 b05415

Conflict of Interest Statement: The authors declare that the research was conducted in the absence of any commercial or financial relationships that could be construed as a potential conflict of interest.

Copyright (c) 2019 Chen, Zhang, Shi, Li, Wang, Song, Xu, Li and Zeng. This is an open-access article distributed under the terms of the Creative Commons Attribution License (CC BY). The use, distribution or reproduction in other forums is permitted, provided the original author(s) and the copyright owner(s) are credited and that the original publication in this journal is cited, in accordance with accepted academic practice. No use, distribution or reproduction is permitted which does not comply with these terms. 